\title{
Evaluation of a training programme for new peer instructors in Healthy Food Made Easy - a cooking for health course for disadvantaged groups
}

\author{
S. Rigney ${ }^{1}$, C. Cunningham ${ }^{2}$ and E. Purtill ${ }^{2}$ \\ ${ }^{1}$ Robert Gordon University, Aberdeen AB251XF, UK and ${ }^{2}$ Community Nutrition and Dietetic Services, Health Services \\ Executive Dublin Mid-Leinster, Tullamore, County Offaly, Ireland
}

People living on a low income have poor diets and high mortality levels from diet-related diseases ${ }^{(1)}$. Lower education levels have also been found to be a contributor to sub-optimal diet ${ }^{(2)}$. The National Adult Literacy Agency states that one in every four adults in Ireland has problems with reading and writing ${ }^{(3)}$. These individuals are targeted for the Healthy Food Made Easy (HFME) programme. HFME is a peer led nutrition education programme designed for disadvantaged groups, aiming at increasing nutritional knowledge, practical cookery skills and at promoting a healthier lifestyle.

The aim of this study was to assess the efficacy of the training module, which was carried out over 6 weeks for new peer educators $(n 6)$, to enable them to deliver the HFME programme. Both qualitative and quantitative methods were used to assess their confidence levels, new skills attainment and attitudinal and behaviour changes.

A short self-report questionnaire was delivered at baseline and post training in order to investigate skills required and concerns over delivery of the programme. Telephone administered questionnaires assessing enjoyment, confidence and new skills required to deliver HFME were completed 6 weeks post training by which time instructors had delivered their first HFME programme. Focus testing was carried out with the instructors $(n 6)$ on the final day of training. A topic guide was used and included questions on satisfaction with the training and appropriateness of teaching methods. The focus groups were recorded and transcribed verbatim, all transcription were analysed manually and themes and topics identified.

All six instructors completed questionnaires and participated in the focus group. All were confident to lead their own HFME group. The number of skills identified as necessary for delivering HFME doubled after the training module - from six to twelve. At baseline, knowledge $(30 \%)$, listening $(20 \%)$ and confidence $(20 \%)$ were deemed the most important skills. Post training this changed to listening $(13 \%)$, knowledge $(13 \%)$, presentation $(13 \%)$, confidence $(10 \%)$ and facilitation $(10 \%)$. In the focus group, the themes that emerged showed that the training course was acceptable and enjoyable with session length and teaching styles being very suitable. The instructors stated that they were more aware of making healthier food choices in their own homes, such as purchasing less convenience food. They also commented that the practical cookery lessons were simple and straight forward and they were confident that they could replicate the recipes in their own HFME programmes.

Satisfaction with the training programme was very high, specifically the practical cookery element and the participatory way that it was delivered. Confidence levels in facilitating a group also rose, mainly in response to the new skills and dietary knowledge attained.

The training programme was effective in delivering competent and confident new peer instructors. The Community Nutrition and Dietetic services will continue to use this model of training in the future for all HMFE instructors.

1. Anderson A (2007) Nutrition interventions in women in low income-groups in the UK. Proc Nutr Soc 66, $25-32$.

2. Freisling H, Emladfa I \& Gall L (2006) The effect of socioeconomic status on dietary intake, physical activity and body mass index in Austrian pregnant women. J Hum Nutr Diet 19, 437-445.

3. NALA (2010) Literacy in Ireland. Dublin: NALA; available at http://www.nala.ie/literacy-ireland 